\title{
Liberação de fósforo e potássio durante a decomposição de resíduos culturais em plantio direto(1)
}

\author{
Sandro José Giacomini(2), Celso Aita ${ }^{(2)}$, André Paulo Hübner ${ }^{(2)}$, Adilson Lunkes ${ }^{(2)}$, \\ Elias Guidini(2) e Elizandro Brum do Amaral(2)
}

\begin{abstract}
Resumo - O objetivo deste trabalho foi avaliar a liberação de $\mathrm{P}$ e $\mathrm{K}$ de resíduos culturais de aveia-preta, ervilhaca comum e nabo forrageiro e de consórcios de aveia e ervilhaca, em plantio direto. Os resíduos culturais foram dispostos em bolsas de nylon com $20 \times 20 \mathrm{~cm}$ e malha de $0,5 \mathrm{~mm}$, as quais foram distribuídas na superfície do solo e coletadas após 15, 29, 43, 59, 71, 82, 112 e 182 dias. Em cada coleta o material foi secado a $65^{\circ} \mathrm{C}$, determinando-se as quantidades remanescentes de matéria seca e a sua concentração em $\mathrm{P}$ e potássio. Aos valores observados para as quantidades remanescentes de $\mathrm{P}(\mathrm{Pr})$ e $\mathrm{K}$ (Kr) foi ajustado o modelo assintótico $\left[\left(\mathrm{Pr}\right.\right.$ e $\left.\mathrm{Kr}=\mathrm{Ae}^{-\mathrm{kt}}+(100-\mathrm{A})\right]$ a partir do qual estimou-se a taxa $(\mathrm{k})$ de liberação de $\mathrm{P}$ e K e a proporção desses nutrientes dos compartimentos lábil (A) e recalcitrante (100 - A) dos resíduos culturais. A taxa de liberação do $\mathrm{K}$ foi 4,5 vezes maior do que a do fósforo. A quantidade de $\mathrm{P}$ remanescente na fase inicial da decomposição foi inversamente proporcional à concentração do $\mathrm{P}$ solúvel em água dos resíduos culturais. Nos resíduos culturais do consórcio aveia + ervilhaca, o P e o K foram liberados mais lentamente do que o observado nessas espécies em cultivo solteiro. Esses resultados indicam um maior potencial de sincronismo entre a demanda de $\mathrm{P}$ e $\mathrm{K}$ pelas culturas comerciais e a liberação desses nutrientes dos resíduos culturais provenientes de consórcios entre aveia e ervilhaca do que dos provenientes do cultivo isolado de ervilhaca.
\end{abstract}

Termos para indexação: Avena strigosa, Vicia sativa, Raphanus sativus, resíduos de cultura.

Phosphorus and potassium release during decomposition of crops residues in no-tillage system

Abstract - The objective of this work was to evaluate the release of $\mathrm{P}$ and $\mathrm{K}$ from residues of single oat, vetch and forage radish and from mixtures of oat and vetch, under no-tillage system. The residues were placed in $0.5 \mathrm{~mm}$ mesh nylon bags $(20 \times 20 \mathrm{~cm})$, which were placed on soil surface and taken out at 15 , $29,43,59,71,82,112$ and 182 days after. At each sampling date the material was dried at $65^{\circ} \mathrm{C}$ and the amount of dry matter remaining and its concentration of $\mathrm{P}$ and potassium were determined. It was adjusted the asymptotic model $\left[\left(\mathrm{Pr}\right.\right.$ and $\left.\mathrm{Kr}=\mathrm{Ae}^{-\mathrm{kt}}+(100-\mathrm{A})\right]$ to observed values for the remaining $\mathrm{P}$ and $\mathrm{K}(\mathrm{Pr}$ and $\mathrm{Kr}$ ) which was used to estimate the rate $(\mathrm{k})$ of $\mathrm{P}$ and $\mathrm{K}$ release and the proportion of these nutrients of the labile (A) and recalcitrant (100 - A) pools of crop residues. The rate of $\mathrm{K}$ release was 4.5 times greater than was for phosphorus. The amount of remaining $\mathrm{P}$ in the initial phase of the decomposition was inversely proportional to the concentration of water soluble $\mathrm{P}$ in the residues. In the residues of the oat + vetch mixture the $\mathrm{P}$ and $\mathrm{K}$ release were slower than the observed for single species. These results indicate a greater potential of synchrony between commercial crops $\mathrm{P}$ and $\mathrm{K}$ demand and $\mathrm{P}$ and $\mathrm{K}$ release from residues of oat + vetch mixture as compared to single vetch.

Index terms: Avena strigosa, Vicia sativa, Raphanus sativus, crop residues.

(1) Aceito para publicação em 17 de julho de 2003 .

Extraído da dissertação de mestrado apresentada pelo primeiro autor à Universidade Federal de Santa Maria (UFSM), Santa Maria, RS. Financiado pela Fapergs, PRONEX-CNPq/FINEP.

(2) UFSM, Centro de Ciências Rurais (CCR), Dep. de Solos, Caixa Postal 221, CEP 97105-900 Santa Maria, RS. Email: sjgiacomini@mail.ufsm.br, caita@ccr.ufsm.br, andrepaulohubner@bol.com.br, adilsonlunkes@mail.ufsm.br, elias@mail.ufsm.br, elizandro@mail.ufsm.br

\section{Introdução}

Na última década, com o avanço da área cultivada sob sistema plantio direto (SPD) na Região Sul do Brasil, aumentou o interesse pelo uso de plantas de cobertura de solo no outono/inverno, com destaque para a ervilhaca comum, a aveia-preta e o nabo forrageiro (Aita, 1997). A maioria dos trabalhos 
realizados tem enfocado o uso dessas espécies em cultivos isolados, e os principais aspectos avaliados envolvem o efeito das espécies na produtividade das culturas comerciais em sucessão, na dinâmica do N no solo e na conservação do solo e da água (Derpsch et al., 1985; Aita, 1997). Pouca atenção, todavia, tem sido dada ao estudo da liberação de $\mathrm{P}$ e de $\mathrm{K}$ dos nutrientes dos resíduos dessas culturas, especialmente quando consorciadas.

A velocidade de liberação de nutrientes dos resíduos culturais durante o processo de decomposição depende da localização e da forma em que esses nutrientes se encontram no tecido vegetal. O potássio, que se encontra em componentes não estruturais e na forma iônica no vacúolo das células das plantas (Marschner, 1995), é rapidamente lixiviado logo após o manejo das plantas de cobertura, com pequena dependência dos processos microbianos. No caso do $\mathrm{P}$, cuja maior parte encontra-se na planta associada a componentes orgânicos do tecido vegetal (Marschner, 1995), sua liberação está intimamente ligada ao processo de decomposição pelos microrganismos do solo.

Os principais fatores que afetam a taxa de mineralização dos compostos orgânicos são as condições edafoclimáticas (temperatura, umidade, $\mathrm{pH}$, teores de $\mathrm{O}_{2}$ e de nutrientes no solo) e a qualidade do substrato (fração solúvel, nutrientes, lignina, polifenóis e as relações $\mathrm{C} / \mathrm{N}$, lignina/N e lignina + polifenóis/N) (Paul \& Clark, 1996). Ao contrário do $\mathrm{N}$, poucos estudos têm sido realizados de modo a relacionar a taxa de mineralização de $\mathrm{P}$ com as características bioquímicas dos resíduos culturais das plantas de cobertura. Quando a relação $\mathrm{C} / \mathrm{P}$ foi relacionada com as taxas de mineralização de $\mathrm{P}$ dos resíduos culturais, os resultados obtidos foram inconsistentes (White \& Ayoub, 1983; Buchanan \& King, 1993; Schomberg \& Steiner, 1999). A concentração em $P$ inorgânico (Pi) pode ser um bom indicador da velocidade de liberação de P nos estágios iniciais de decomposição dos resíduos culturais, pois o Pi é solúvel em água e pode representar de $15 \%$ do $\mathrm{P}$ total das plantas deficientes em $\mathrm{P}$ a até $70 \%$ em plantas com teor próximo ao limite de toxidez (Marschner, 1995; Malavolta et al., 1997).
Nos últimos anos, tem aumentado o número de trabalhos envolvendo o uso de plantas de cobertura em consórcio, principalmente entre leguminosas e não-leguminosas, com o objetivo de avaliar o potencial desta estratégia de cultivo em aumentar a produção de fitomassa e favorecer o fornecimento de $\mathrm{N}$ às culturas em sucessão, especialmente o milho (Clark et al., 1994; Giacomini, 2001). Esta prática também deverá ter reflexos sobre a ciclagem de $\mathrm{P}$ e K em sistemas agrícolas com o uso de plantas de cobertura de solo, principalmente em sistema plantio direto.

O objetivo deste trabalho foi avaliar a liberação de $\mathrm{P}$ e de $\mathrm{K}$ de resíduos culturais provenientes de cultivos isolados de aveia-preta, ervilhaca e nabo forrageiro e de consórcios entre aveia e ervilhaca.

\section{Material e Métodos}

O experimento foi realizado a campo, de outubro de 1999 a março de 2000, na área experimental do Departamento de Solos da Universidade Federal de Santa Maria, Santa Maria, RS, em Argissolo Vermelho distrófico arênico (Embrapa, 1999) textura franco-arenosa no horizonte A e franco-argilosa no horizonte B. O clima da região é subtropical úmido, tipo $\mathrm{Cfa}$, conforme classificação de Köppen.

A avaliação da liberação de $\mathrm{P}$ e de $\mathrm{K}$ foi feita em resíduos culturais de ervilhaca comum (EC) (Vicia sativa L.), de nabo forrageiro (NF) (Raphanus sativus L. ) e de aveiapreta (AP) (Avena strigosa Schieb), provenientes de experimento em que essas espécies foram cultivadas a campo, em sistema isolado e em consórcio, compondo os seguintes tratamentos: $100 \% \mathrm{AP}\left(80 \mathrm{~kg} \mathrm{ha}^{-1}\right.$ de sementes), $100 \%$ EC $\left(80 \mathrm{~kg} \mathrm{ha}^{-1}\right), 100 \%$ NF $\left(14 \mathrm{~kg} \mathrm{ha}^{-1}\right), 15 \%$ AP $+85 \%$ EC e $45 \%$ AP $+55 \%$ EC. As quantidades de sementes de cada espécie são relativas a poder germinativo de $100 \%$.

No pleno florescimento da aveia, no início do florescimento da ervilhaca e no final do florescimento do nabo forrageiro, coletaram-se duas subamostras de $0,36 \mathrm{~m}^{2}$ por parcela de cada tratamento para formar uma amostra composta. Nos tratamentos em consórcio, as espécies foram separadas para determinar sua contribuição na produção total de matéria seca (MS) nos tratamentos. A aveia foi separada em colmos, folhas e panículas; a ervilhaca, em talos e folhas; e o nabo forrageiro, em hastes, folhas e inflorescências. Na determinação da matéria seca, cada constituinte foi secado em ambiente protegido $\left(25^{\circ}\right.$ a $\left.30^{\circ} \mathrm{C}\right)$ até peso constante. 
A dinâmica de liberação do $\mathrm{P}$ e do $\mathrm{K}$ dos resíduos culturais das plantas de cobertura foi monitorada a campo por meio de bolsas de nylon com 20x20 cm e malha de $0,5 \mathrm{~mm}$. Os constituintes das plantas foram cortados manualmente em pedaços de aproximadamente $19 \mathrm{~cm}$ antes de serem colocados nas bolsas. A quantidade de material colocado nas bolsas foi equivalente à matéria seca produzida por espécie nos diferentes tratamentos, obedecendo-se à proporção entre os diferentes constituintes de cada espécie. Imediatamente após a colocação dos resíduos culturais nas bolsas, utilizaram-se quatro repetições de cada tratamento para determinar a quantidade inicial de matéria seca a $65^{\circ} \mathrm{C}$, em estufa, até peso constante. A seguir, o material foi pesado e moído em moinho Willey equipado com peneira de 40 mesh e analisado quanto às concentrações de C, P e K, conforme Tedesco et al. (1995), e lignina, conforme Soest \& Wine (1967). A fração solúvel foi extraída em água fria $\left(21^{\circ} \mathrm{C}\right)$, conforme Aita (1996), determinando-se a concentração em P solúvel em água (Psa) (Murphy \& Riley, 1962) e K solúvel em água (Ksa) (Tedesco et al., 1995). Algumas características dos resíduos culturais são apresentadas na Tabela 1.

Em 23 de outubro de 1999 foram distribuídas, na superfície do solo, oito bolsas de decomposição com quatro repetições de cada tratamento. As 32 bolsas foram arranjadas em delineamento experimental de blocos ao acaso e as amostragens realizadas aos $15,29,43,59,71,82,112 \mathrm{e}$ 182 dias após a colocação no campo, retirando-se uma bolsa por repetição de cada tratamento. Para quantificar a matéria seca remanescente, os resíduos vegetais de cada coleta foram submetidos à secagem em estufa a $65^{\circ} \mathrm{C}$ até peso constante. Após a moagem, determinaram-se as concentrações de $\mathrm{P}$ e de $\mathrm{K}$ na matéria seca remanescente de cada coleta, conforme Tedesco et al. (1995).

Os dados relativos à precipitação pluvial acumulada e à temperatura média do ar nos intervalos de tempo das diferentes coletas realizadas são apresentados na Figura 1.
Aos valores obtidos das quantidades remanescentes de P e de K em cada coleta foi ajustado o modelo assintótico (Wieder \& Lang, 1982), definido pela equação: $\mathrm{Pr}$ e $\mathrm{Kr}=\mathrm{Ae}^{-\mathrm{kt}}+(100-\mathrm{A})$,

em que $\mathrm{Pr}$ e $\mathrm{Kr}$ são os valores de $\mathrm{P}$ e $\mathrm{K}$ remanescentes (\% da quantidade inicial) no tempo t; A é a quantidade de $\mathrm{P}$ ou $\mathrm{K}$ (\% da quantidade inicial) contida no compartimento lábil (mais facilmente mineralizável); (100 - A) é o $\mathrm{P}$ ou K (\% da quantidade inicial) contido no compartimento recalcitrante (mais difícil mineralização); $\mathrm{k}$ é a taxa constante de liberação de $\mathrm{P}$ e $\mathrm{K}$ do compartimento A.

A partir dos valores da constante de liberação de $\mathrm{P}$ e de $\mathrm{K}$ do compartimento A, calculou-se o tempo de meia-vida $\left(\mathrm{t}_{1 / 2}=0,693 / \mathrm{k}\right)$, conforme Paul \& Clark (1996). O t $\mathrm{t}_{1 / 2}$ é o tempo necessário para que $50 \%$ do $\mathrm{P}$ e do $\mathrm{K}$ daquele compartimento sejam liberados.

Com base no modelo ajustado para as quantidades remanescentes de $\mathrm{P}$ e de $\mathrm{K}$ em cada tratamento, estimou-se a liberação cumulativa desses nutrientes durante o período de avaliação multiplicando-se a porcentagem de liberação do nutriente em cada coleta, obtida mediante o modelo, pelas quantidades iniciais de $\mathrm{P}$ e de $\mathrm{K}\left(\mathrm{kg} \mathrm{ha}^{-1}\right)$ adicionadas inicialmente nas bolsas de decomposição (Tabela 1).

Os resultados referentes às variáveis do modelo ajustado (compartimento A, taxas de liberação de $\mathrm{P}$ e $\mathrm{K}$ e $\mathrm{t}_{1 / 2}$ ) foram submetidos à análise de variância, e as médias comparadas pelo teste Tukey a $5 \%$ de probabilidade.

\section{Resultados e Discussão}

A cinética de liberação de $\mathrm{P}$ dos resíduos culturais diferiu entre os tratamentos (Figura 2 e Tabela 2). Enquanto na ervilhaca, menos de $60 \%$ do $\mathrm{P}$ permaneceu nos resíduos culturais nos primeiros 15 dias, no nabo e na aveia este valor foi superior a $90 \%$. Na segunda amostragem, realizada aos

Tabela 1. Características químicas iniciais dos resíduos culturais de aveia-preta (AP), ervilhaca comum (EC) e nabo forrageiro (NF) e quantidades adicionadas em cada tratamento de matéria seca (MS), fósforo (P), potássio (K), fósforo solúvel em água (Psa) e potássio solúvel em água (Ksa).

\begin{tabular}{|c|c|c|c|c|c|c|c|c|c|c|c|c|c|}
\hline \multirow[t]{2}{*}{ Tratamento } & $\mathrm{C}^{(1)}$ & $\bar{P}$ & $\overline{\mathrm{K}}$ & Psa & Ksa & $\overline{\mathrm{LIG}^{(1)}}$ & \multirow{2}{*}{$\bar{C} / \mathrm{P}$} & \multirow[t]{2}{*}{ LIG/P } & \multirow{2}{*}{\multicolumn{3}{|c|}{$\begin{array}{llc}\text { MS } & P & \mathrm{~K} \\
& - & - \\
& \left.-\mathrm{kg} \mathrm{ha}^{-1}\right)\end{array}$}} & Psa & $\overline{\mathrm{Ksa}}$ \\
\hline & \multicolumn{6}{|c|}{ ( $\left.\mathrm{g} \mathrm{kg}^{-1} \mathrm{de} \mathrm{MS}\right)$} & & & & & & & \\
\hline $100 \% \mathrm{AP}$ & 443 & 2,88 & 21,4 & 1,81 & 16,0 & 66,5 & 154 & 23,1 & 4.390 & 12,6 & 94 & 7,9 & $\overline{70}$ \\
\hline $100 \% \mathrm{EC}$ & 432 & 4,17 & 27,8 & 2,30 & 15,6 & 83,4 & 104 & 20,0 & 2.660 & 11,1 & 74 & 6,1 & 41 \\
\hline $100 \% \mathrm{NF}$ & 416 & 3,21 & 23,9 & 1,64 & 17,4 & 92,1 & 130 & 28,7 & 3.720 & 11,9 & 89 & 6,1 & 65 \\
\hline $15 \% \mathrm{AP}+85 \% \mathrm{EC}^{(2)}$ & 448 & 3,77 & 23,9 & 2,26 & 16,5 & 81,4 & 119 & 21,6 & 3.490 & 13,2 & 84 & 7,9 & 58 \\
\hline $45 \% \mathrm{AP}+55 \% \mathrm{EC}^{(3)}$ & 425 & 3,03 & 24,9 & 2,12 & 17,5 & 78,3 & 140 & 25,8 & 4.240 & 13,8 & 106 & 9,0 & 74 \\
\hline
\end{tabular}

${ }^{(1)} \mathrm{C}$ : carbono; LIG: lignina. ${ }^{(2)} 1,12 \mathrm{Mg} \mathrm{ha}^{-1}$ de MS de aveia $+2,37 \mathrm{Mg}$ ha $^{-1}$ de MS de ervilhaca. ${ }^{(3)} 2,16 \mathrm{Mg} \mathrm{ha}^{-1}$ de MS de aveia $+2,08 \mathrm{Mg}^{-1}$ de MS de ervilhaca. 
29 dias, o P remanescente decresceu para $40 \%$ da quantidade inicial de $\mathrm{P}$ na ervilhaca e para $70 \%$ na aveia. Esses resultados confirmam os obtidos por Da Ros (1993), que avaliou a liberação de P durante a decomposição de diversas plantas de cobertura e verificou que $64 \%$ do $\mathrm{P}$ da ervilhaca e $33 \%$ do $\mathrm{P}$ da aveia foram liberados nos primeiros 30 dias após o manejo das plantas com uma gradagem. Tais resultados podem ser explicados pela maior concentração de P solúvel em água (Psa) da ervilhaca, que superou a aveia em 27\% (Tabela 1). Pois, segundo

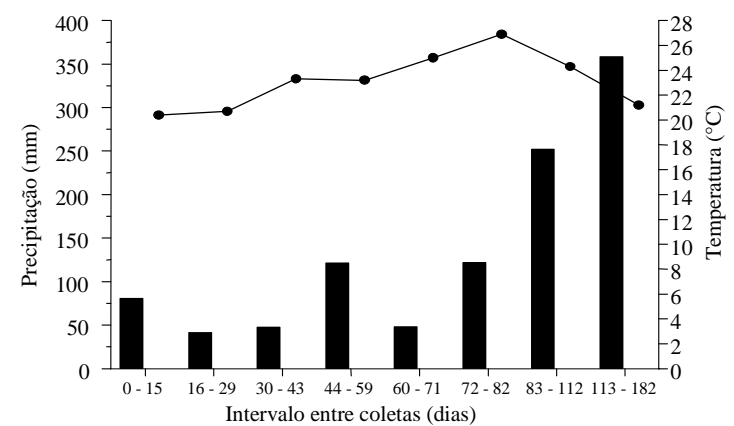

Figura 1. Precipitação pluvial (barras) e temperatura média do ar (linha) em cada intervalo de coleta das bolsas de decomposição com resíduos culturais de plantas de cobertura, durante o período em que permaneceram no campo.
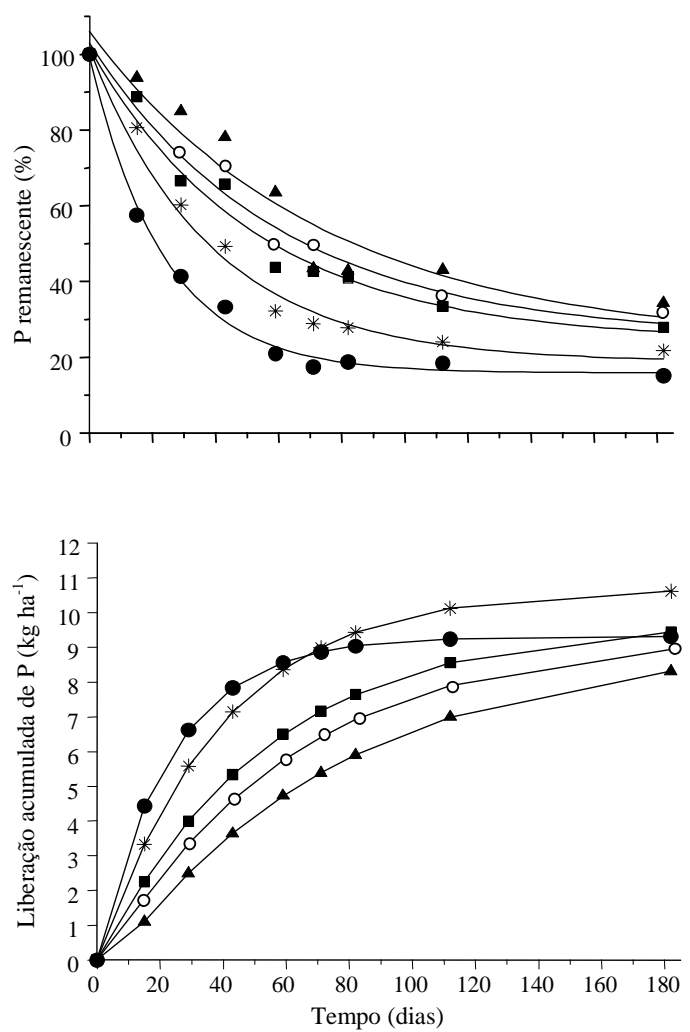

Figura 2. Fósforo remanescente e liberação acumulada de $\mathrm{P}$ durante a decomposição dos resíduos culturais das plantas de cobertura em cultivo isolado e em consórcio (O: $100 \%$ de aveia; $: 100 \%$ de ervilhaca; $\boldsymbol{\Delta}: 100 \%$ de nabo; $*$ : $15 \%$ de aveia $+85 \%$ de ervilhaca; $\mathbf{\square}: 45 \%$ de aveia $+55 \%$ de ervilhaca).

Tabela 2. Parâmetros do modelo ajustado aos valores medidos de $\mathrm{Pe} \mathrm{K}$ remanescentes, tempo de meia-vida $\left(\mathrm{t}_{1 / 2}\right)$ do $\mathrm{P}$ e do $\mathrm{K}$ do compartimento lábil (A) e coeficiente de determinação $\left(\mathrm{R}^{2}\right)$ para o ajuste do modelo em cada tratamento ${ }^{(1)}$.

\begin{tabular}{|c|c|c|c|c|}
\hline Tratamento $^{(2)}$ & $\begin{array}{c}\text { A } \\
(\%) \\
\end{array}$ & $\begin{array}{c}\mathrm{k} \\
\left(\mathrm{dia}^{-1}\right) \\
\end{array}$ & $\begin{array}{l}\mathrm{t}_{1 / 2} \mathrm{~A} \\
\text { (dias) }\end{array}$ & $\mathrm{R}^{2}$ \\
\hline \multicolumn{5}{|c|}{ P remanescente $(\mathrm{Pr})^{(3)}$} \\
\hline $100 \%$ AP & $79,3 \mathrm{a}$ & $0,01683 \mathrm{c}$ & $41,2 \mathrm{ab}$ & $0,97 *$ \\
\hline $100 \% \mathrm{EC}$ & $83,5 \mathrm{a}$ & $0,04252 \mathrm{a}$ & $6,5 d$ & $0,99^{*}$ \\
\hline $100 \% \mathrm{NF}$ & $82,8 \mathrm{a}$ & $0,01355 \mathrm{c}$ & $51,2 \mathrm{a}$ & $0,92 *$ \\
\hline $15 \% \mathrm{AP}+85 \% \mathrm{EC}$ & $82,6 a$ & $0,02600 \mathrm{~b}$ & $26,6 \mathrm{~cd}$ & $0,99 *$ \\
\hline $45 \% \mathrm{AP}+55 \% \mathrm{EC}$ & $77,6 \mathrm{a}$ & $0,01893 \mathrm{c}$ & $36,6 b c$ & $0,97 *$ \\
\hline \multicolumn{5}{|c|}{ K remanescente $(\mathrm{Kr})^{(3)}$} \\
\hline $100 \% \mathrm{AP}$ & $96,6 a$ & $0,05558 \mathrm{~d}$ & $12,5 \mathrm{a}$ & $0,99^{*}$ \\
\hline $100 \% \mathrm{EC}$ & $98,1 \mathrm{a}$ & $0,16748 \mathrm{a}$ & $4,2 \mathrm{c}$ & $0,99^{*}$ \\
\hline $100 \% \mathrm{NF}$ & $96,6 a$ & $0,14025 b$ & $4,9 \mathrm{bc}$ & $0,99 *$ \\
\hline $15 \% \mathrm{AP}+85 \% \mathrm{EC}$ & $97,8 \mathrm{a}$ & $0,09668 \mathrm{c}$ & $7,2 \mathrm{~b}$ & $0,99 *$ \\
\hline $45 \% \mathrm{AP}+55 \% \mathrm{EC}$ & $97,8 \mathrm{a}$ & $0,06856 \mathrm{~d}$ & $10,1 \mathrm{a}$ & $0,99 *$ \\
\hline
\end{tabular}

${ }^{(1)}$ Médias seguidas da mesma letra, na coluna, não diferem entre si pelo teste de Tukey, a 5\% de probabilidade. (2)AP: aveia-preta; EC: ervilhaca comum; NF: nabo forrageiro. ${ }^{(3)}$ Modelo para $\mathrm{Pr}$ e $\mathrm{Kr}=\mathrm{Ae}^{(-\mathrm{kt})}+(100-\mathrm{A})$, onde $\mathrm{A}$ : $\mathrm{Pe} \mathrm{K}$ (\% da quantidade inicial) contidos no compartimento mais facilmente mineralizável; k: taxa constante de liberação de P e de K do compartimento A; t: tempo; (100 - A): P e K (\% da quantidade inicial) contidos no compartimento de mais difícil mineralização (recalcitrante). *Significativo a $5 \%$ de probabilidade. 
Buchanan \& King (1993), a rápida liberação de P no período inicial da decomposição dos resíduos culturais está ligada à perda do $\mathrm{P}$ solúvel em água.

A importância da concentração de Psa na liberação de $\mathrm{P}$ durante a decomposição ficou evidenciada quando se avaliou a correlação linear entre as quantidades remanescentes de $\mathrm{P}$ e alguns constituintes dos resíduos culturais das plantas de cobertura, em três datas de amostragem (Tabela 3). O Psa foi o constituinte que melhor se correlacionou negativamente com as quantidades remanescentes de $\mathrm{P}$ dos resíduos nos primeiros 29 dias, evidenciando a importância em se conhecer a concentração inicial dessa fração de $\mathrm{P}$ a fim de utilizá-la como indicador da velocidade de liberação de $\mathrm{P}$ dos resíduos culturais na fase inicial de decomposição. Após o primeiro mês de decomposição, a porcentagem de $\mathrm{P}$ remanescente esteve mais relacionada à concentração de $\mathrm{P}$ total nos resíduos culturais. $\mathrm{O}$ teor em lignina e as relações $\mathrm{C} / \mathrm{P}$ e lignina/ $\mathrm{P}$ foram os atributos que apresentaram a mais baixa correlação com o P remanescente nas três amostragens (Tabela 3 ).

A maior parte do $\mathrm{P}$ do tecido vegetal encontra-se no vacúolo da célula, na forma mineral, bastante solúvel em água (Marschner, 1995). Para que ele seja liberado dos resíduos culturais o vacúolo deve ser rompido. Considerando que durante os primeiros 29 dias após a colocação das bolsas de decomposição no campo a chuva acumulada atingiu cerca de $120 \mathrm{~mm}$ (Figura 1), é provável que, neste período, a maior parte do Psa, constituído principalmente por $\mathrm{P}$ inorgânico (Pi) e monoésteres, tenha sido lixiviada dos resíduos culturais das plantas de cobertura, restando nos resíduos as formas de $\mathrm{P}$ não solúvel em água (maioria diésteres: ácidos nucléicos, fosfolipídios, fosfoproteínas), dependentes da po- pulação microbiana do solo para a sua mineralização (Frossard et al., 1995).

Em condições de chuva simulada, Miller et al. (1994) verificaram relação estreita entre a liberação de $\mathrm{P}$ de resíduos culturais de plantas de cobertura e o seu teor em P total. Segundo esses autores, logo após uma chuva de $30 \mathrm{~mm}$, o conteúdo de $\mathrm{P}$ total diminuiu em 33\% nos resíduos culturais de azevém (Lolium multiflorum L.), $20 \%$ no trevo-encarnado (Trifolium incarnatum L.) e $32 \%$ no nabo (Raphanus sativus L. var. oleiferus Metzg.). A maior porcentagem de diminuição do $\mathrm{P}$ total no azevém e no nabo foi atribuída ao fato de estas espécies terem apresentado uma concentração de $\mathrm{P}$ no tecido vegetal de $20 \%$ e $167 \%$ superior ao do trevo-encarnado, respectivamente. Entretanto, no presente estudo, o teor de $\mathrm{P}$ total das espécies foi bom indicador da liberação de $\mathrm{P}$ apenas após a fase inicial de decomposição dos resíduos culturais (Tabela 3).

O consórcio entre aveia e ervilhaca aumentou a quantidade remanescente de $\mathrm{P}$ nos resíduos culturais, em relação à leguminosa isolada (Figura 2). Nos primeiros 29 dias, o P remanescente foi de aproximadamente $40 \%$ na ervilhaca isolada, aumentando para $60 \%$ no consórcio com $15 \% \mathrm{AP}+85 \% \mathrm{EC}$, e para $67 \%$ no consórcio com $45 \% \mathrm{AP}+55 \%$ ervilhaca comum. A liberação mais gradual do $\mathrm{P}$ dos resíduos culturais dos consórcios em relação à ervilhaca isolada, na fase inicial de decomposição, deverá contribuir para aumentar a eficiência do $\mathrm{P}$ fornecido pelas plantas de cobertura às culturas em sucessão, pelo fato de se aproximar melhor da curva de absorção de $\mathrm{P}$ pelas culturas, diminuindo tanto a sua imobilização pela população microbiana como a sua fixação no solo pelos óxidos de ferro (Büll, 1993; Frossard et al., 1995).

Tabela 3. Coeficientes de correlação linear entre o fósforo remanescente e algumas características dos resíduos culturais de aveia preta, ervilhaca comum e nabo forrageiro, nas coletas realizadas aos 29, 82 e 182 dias após a colocação das bolsas de decomposição no campo ${ }^{(1)}$.

\begin{tabular}{llllll}
\hline P remanescente & \multicolumn{1}{c}{$\mathrm{P}$} & Psa & LIG & C/P & LIG/P \\
\hline 29 dias & $-0,815^{\mathrm{ns}}$ & $-0,903^{* *}$ & $-0,070^{\mathrm{ns}}$ & $0,707^{\mathrm{ns}}$ & $0,874^{*}$ \\
82 dias & $-0,959^{* * *}$ & $-0,845^{\mathrm{ns}}$ & $-0,807^{\mathrm{ns}}$ & $0,866^{*}$ & $0,850^{\mathrm{ns}}$ \\
182 dias & $-0,903^{* *}$ & $-0,903^{* *}$ & $-0,076^{\mathrm{ns}}$ & $0,814^{\mathrm{ns}}$ & $0,850^{\mathrm{ns}}$ \\
\hline
\end{tabular}

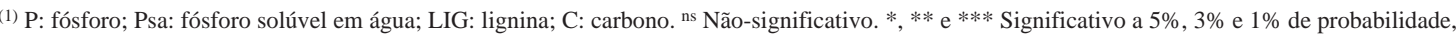
respectivamente. 
$\mathrm{O}$ modelo ajustado às quantidades remanescentes de $\mathrm{P}$ revelou que $77,6 \%$ a $83,5 \%$ do total de $\mathrm{P}$ adicionado ao solo pelos resíduos culturais das plantas de cobertura estavam contidos no compartimento de P mais facilmente mineralizável, não havendo diferenças entre as plantas de cobertura nesse sentido (Tabela 2). Entre as culturas isoladas, a ervilhaca foi a que apresentou a maior constante de liberação de $\mathrm{P}\left(\mathrm{k}=0,04252 \mathrm{dia}^{-1}\right)$, com um tempo de meiavida desse elemento de aproximadamente seis vezes inferior à aveia e oito vezes inferior ao nabo, os quais não diferiram entre si. Tais resultados se devem, provavelmente, à presença de maiores concentrações de Pi na leguminosa do que na aveia e no nabo.

Como o nabo possui baixa relação C/N (Aita, 1997), era de se esperar que o $P$ do compartimento mais facilmente mineralizável fosse liberado mais rapidamente do que na aveia. $\mathrm{O}$ fato de o tempo de meia-vida $\left(\mathrm{t}_{1 / 2}\right)$ do $\mathrm{P}$ não ter diferido do tempo na aveia se deve, provavelmente, ao estádio de desenvolvimento em que o nabo se encontrava quando foi coletado para ser colocado nas bolsas de decomposição. Como o nabo estava no final do período de floração, a maior parte das folhas já havia caído ao solo, aumentando a proporção de hastes em relação às folhas. É provável que o $\mathrm{P}$ contido nas hastes seja liberado mais lentamente do que o $\mathrm{P}$ presente nas folhas e que, portanto, o nabo manejado na fase de florescimento apresente um padrão de liberação de $\mathrm{P}$ mais próximo ao da ervilhaca do que o verificado na aveia.

Com o aumento na proporção de aveia nos consórcios com ervilhaca, não houve alteração na quantidade de $\mathrm{P}$ presente no compartimento mais facilmente mineralizável em relação à leguminosa isolada, porém houve redução significativa na taxa constante $(\mathrm{k})$ de liberação do $\mathrm{P}$ dos resíduos culturais deste compartimento (Tabela 2). Quando a proporção de aveia foi de $15 \%$, o valor de $\mathrm{k}$ foi aproximadamente $40 \%$ menor do que o da ervilhaca isolada, e o tempo de meia-vida do $\mathrm{P}$ desse compartimento aumentou de 6,5 para 26,6 dias. No consórcio com $45 \%$ de aveia, o valor de $\mathrm{k}$ foi $55 \%$ menor e o $\mathrm{t}_{1 / 2}$ passou para 36,6 dias.

Seis meses após a distribuição das bolsas de decomposição na superfície do solo, as quantidades remanescentes de $\mathrm{P}$ nos resíduos culturais das espécies isoladas foram de $15 \%$ na ervilhaca, $34 \%$ no nabo e $32 \%$ na aveia. Nos consórcios, as quantidades remanescentes de $\mathrm{P}$ apresentaram valores intermediários aos observados nas culturas de aveia e ervilhaca isoladas (Figura 2).

A liberação acumulada de $\mathrm{P}$ dos resíduos culturais variou nos primeiros 15 dias, de aproximadamente $1,0 \mathrm{~kg} \mathrm{ha}^{-1}$ no nabo a $4,5 \mathrm{~kg} \mathrm{ha}^{-1}$ na ervilhaca isolada (Figura 2). Ao final do período avaliado, o tratamento com $15 \% \mathrm{AP}+85 \%$ EC foi o que liberou maiores quantidades de $\mathrm{P}\left(10,6 \mathrm{~kg} \mathrm{ha}^{-1}\right)$, seguido dos tratamentos com a ervilhaca isolada e o consórcio $45 \% \mathrm{AP}+55 \% \mathrm{EC}$, com cerca de $9,4 \mathrm{~kg} \mathrm{ha}^{-1}$ de $\mathrm{P}$ liberados, e da aveia e nabo isolados, cuja liberação média de $\mathrm{P}$ atingiu $8,6 \mathrm{~kg} \mathrm{ha}^{-1}$. Apesar de o consórcio com $15 \%$ de aveia ter apresentado taxa de liberação de P $40 \%$ menor do que a da ervilhaca isolada, a quantidade de $\mathrm{P}$ liberada foi maior. Tais resultados devem-se ao fato de que, além das taxas de decomposição, a quantidade total de nutrientes liberada depende da quantidade de nutrientes acumulada na fitomassa das plantas de cobertura. No presente trabalho, o consórcio com $15 \%$ de aveia acumulou cerca de $19 \%$ mais $\mathrm{P}$ do que a ervilhaca isolada (Tabela 1).

O K foi rapidamente liberado dos resíduos culturais (Figura 3), com taxa média de liberação 4,5 vezes maior do que a observada em relação ao $\mathrm{P}$ (Tabela 2). Na primeira amostragem, realizada aos 15 dias, cerca de $10 \%$ do $\mathrm{K}$ da ervilhaca e $15 \%$ do $\mathrm{K}$ do nabo permaneciam nos resíduos culturais dessas espécies. Nesse mesmo período, o K remanescente da aveia era de aproximadamente $45 \%$. Com a participação da gramínea nos consórcios com ervilhaca, a liberação desse elemento dos tratamentos foi intermediária às das duas culturas isoladas.

A rápida liberação de $\mathrm{K}$ ocorrida nos primeiros 15 dias confirma os resultados de Da Ros (1993) e Schomberg \& Steiner (1999), e pode ser atribuída ao fato de o K ser um elemento que não está associado a nenhum componente estrutural do tecido vegetal (Marschner, 1995). Schomberg \& Steiner (1999) observaram que, com precipitações elevadas, houve maior liberação de K nas leguminosas do que nas gramíneas. Segundo esses autores, mecanismos físicos (chuva) e químicos (qualidade do resíduo) 
estão envolvidos na liberação do nutriente e que, em razão da elevada taxa de liberação de $\mathrm{K}$ dos resíduos culturais, parte dele poderá ser perdido no solo pela lixiviação. Nesse sentido, o K talvez seja o elemento cuja redução do prazo de implantação das culturas em sucessão seja mais importante para minimizar essas perdas.

Aproximadamente $70 \%$ do $\mathrm{K}$ no tecido vegetal das diferentes espécies é solúvel em água (Ksa) (Tabela 1). Portanto, com a ocorrência das chuvas após o manejo das espécies, a maior parte desse elemento
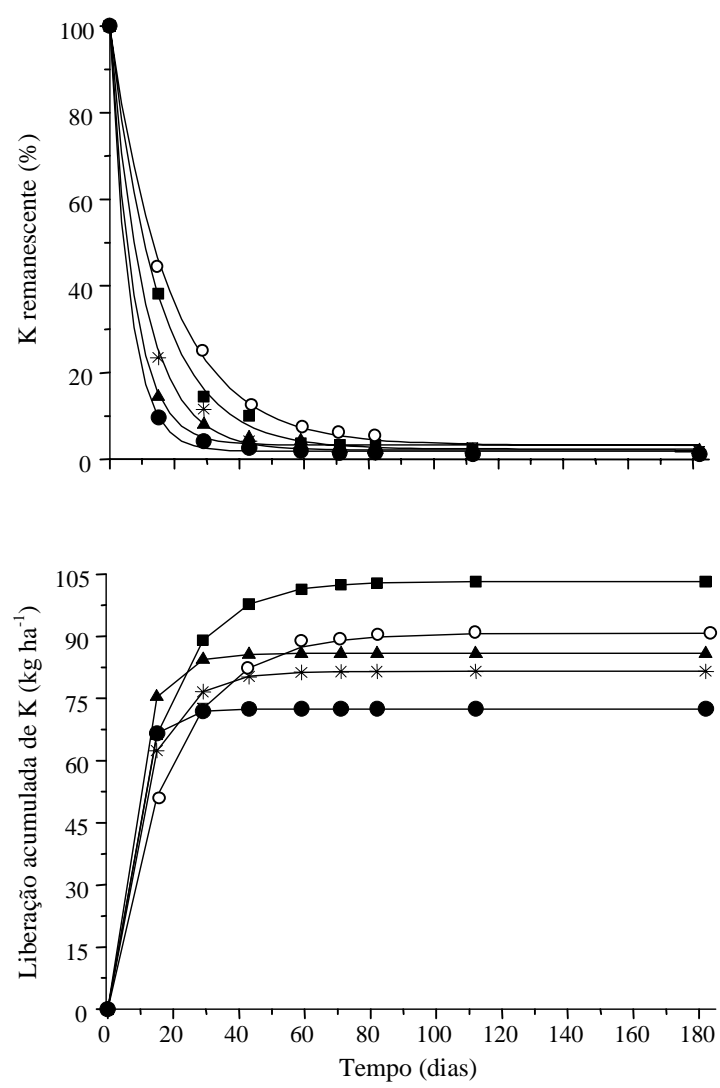

Figura 3. Potássio remanescente e liberação acumulada de $\mathrm{K}$ durante a decomposição dos resíduos culturais das plantas de cobertura em cultivo isolado e em consórcio (○: $100 \%$ de aveia; $100 \%$ de ervilhaca; $\boldsymbol{\Delta}: 100 \%$ de nabo; $*$ : $15 \%$ de aveia $+85 \%$ de ervilhaca; $\mathbf{\square}$ : $45 \%$ de aveia $+55 \%$ de ervilhaca). foi liberada dos resíduos culturais para o solo. A rápida liberação do $\mathrm{K}$ do tecido vegetal resultou num tempo de meia-vida $\left(\mathrm{t}_{1 / 2}\right)$ de 13 dias na aveia e de apenas quatro dias na ervilhaca, a qual não diferiu do nabo (Tabela 2).

O padrão de liberação de $\mathrm{K}$ dos resíduos culturais da ervilhaca e do nabo isolados e do consórcio $15 \%$ AP $+85 \%$ EC foi semelhante, e praticamente todo o $\mathrm{K}$ foi liberado nos primeiros 29 dias (Figura 3). Nos tratamentos com aveia isolada e no consórcio 45\% AP + 55\% EC a liberação foi mais lenta, estabilizando-se a partir de 60 dias. Essa redução na velocidade de liberação do $\mathrm{K}$, pelo aumento da aveia nos consórcios com ervilhaca, pode ter reflexos importantes na diminuição das perdas do nutriente no solo, especialmente naqueles com textura arenosa e com baixa capacidade de troca de cátions, onde o nutriente poderá ser lixiviado. Além da liberação mais lenta, as quantidades totais de $\mathrm{K}$ liberadas em seis meses nos tratamentos com $45 \% \mathrm{AP}+55 \% \mathrm{EC} \mathrm{e}$ aveia isolada foram superiores à observada em ervilhaca isolada em $25 \%$ e $42 \%$, respectivamente (Figura 3).

A quantidade total de $\mathrm{K}$ liberado dos resíduos culturais da gramínea isolada, após seis meses, foi de $91 \mathrm{~kg} \mathrm{ha}^{-1}$ contra apenas $73 \mathrm{~kg} \mathrm{ha}^{-1}$ dos resíduos culturais da leguminosa isolada. Com o nabo forrageiro, a quantidade de K liberada foi de $86 \mathrm{~kg} \mathrm{ha}^{-1}$. Nesse mesmo período, os resíduos culturais provenientes dos consórcios com $15 \%$ e $45 \%$ de aveia na mistura com a ervilhaca liberaram aproximadamente $81 \mathrm{~kg} \mathrm{ha}^{-1}$ e $103 \mathrm{~kg} \mathrm{ha}^{-1}$ de $\mathrm{K}$, respectivamente.

\section{Conclusões}

1. O consórcio de aveia e ervilhaca reduz a taxa constante de liberação do $\mathrm{P}$ e do $\mathrm{K}$ dos resíduos culturais em relação à ervilhaca isolada.

2. A concentração de P solúvel em água das plantas de cobertura pode ser usada como indicador da velocidade de liberação de $\mathrm{P}$ durante a fase inicial de decomposição dos resíduos culturais.

3. A maior parte do $\mathrm{K}$ dos resíduos culturais das plantas de cobertura é liberada logo após o manejo das espécies. 


\section{Referências}

AITA, C. Couplage des cycles du carbone et de l'azote dans les sols cultivés: étude au champ des processus de décomposition après apport de matière organique fraîche. 1996. 196 f. Thèse (Doctorat en Sciences de la Terre) Institut National de la Recherche Agronomique, Université de Paris VI, Paris, 1996.

AITA, C. Dinâmica do nitrogênio no solo durante a decomposição de plantas de cobertura: efeito sobre a disponibilidade de nitrogênio para a cultura em sucessão. In: FRIES, M. R.; DALMOLIN, R. S. D. Atualização em recomendação de adubação e calagem: ênfase em plantio direto. Santa Maria: Pallotti, 1997. p. 76-111.

BUCHANAN, M.; KING, L. Carbon and phosphorus losses from decomposing crop residues in no till and conventional till agroecosystems. Agronomy Journal, Madison, v. 85, n. 3, p. 631-638, 1993.

BÜLL, L. T. Nutrição mineral do milho. In: BÜLL, L. T.; CANTARELLA, H. (Org.). Cultura do milho: fatores que afetam a produtividade. Piracicaba: Potafos, 1993. p. 63-146.

CLARK, A. J.; DECKER, A. M.; MEISINGER, J. J. Seeding rate and kill date effects on hairy vetch-cereal rye cover crop mixtures for corn production. Agronomy Journal, Madison, v. 86, n. 6, p. 1065-1070, 1994

DA ROS, C. O. Plantas de inverno para cobertura do solo e fornecimento de nitrogênio ao milho em plantio direto. 1993. 85 f. Dissertação (Mestrado em Agronomia) Universidade Federal de Santa Maria, Santa Maria, 1993.

DERPSCH, R.; SIDIRAS, N.; HEINZMANN, F. X. Manejo do solo com coberturas verdes de inverno. Pesquisa Agropecuária Brasileira, Brasília, v. 20, n. 7, p. 761-773, jul. 1985.

EMBRAPA. Centro Nacional de Pesquisa de Solos (Rio de Janeiro, RJ). Sistema brasileiro de classificação de solos. Brasília: Embrapa-SPI/Embrapa-CNPS, 1999. $412 \mathrm{p}$.

FROSSARD, E.; FROSSARD, M.; HEDLEY, M. J.; MATHERELL, A. Reactions controlling the cycling of $\mathrm{P}$ in soil. In: TIESSNM, H. (Org.). Phosphorus in the global environment: transfers, cycles and management. Chichester: J. Wiley, 1995. p. 107-146.
GIACOMINI, S. J. Consorciação de plantas de cobertura no outono/inverno e fornecimento de nitrogênio ao milho em sistema plantio direto. 2001. 124 f. Dissertação (Mestrado em Agronomia) - Universidade Federal de Santa Maria, Santa Maria, 2001.

MALAVOLTA, E.; VITTI, G. C.; OLIVEIRA, S. A. Avaliação do estado nutricional das plantas: princípios e aplicações. 2. ed. Piracicaba: Potafos, 1997. 319 p.

MARSCHNER, H. Functions of mineral nutrients: macro-nutrients. In: MINERAL nutrition of higher plants. $2^{\text {nd }}$ ed. San Diego: Academic, 1995. p. 229-312.

MILLER, M. H.; BEAUCHAMP, E. G.; LAUZON, J. D. Leaching of nitrogen phosphorus from the biomass of three cover crop species. Journal of Environmental Quality, Madison, v. 23, n. 2, p. 267-272, 1994.

MURPHY J.; RILEY J. P. A modified single solution method for determination of phosphate in natural waters. Analytica Chimica Acta, Amsterdam, v. 26, n. 1, p. 31-36, 1962

PAUL, E. A.; CLARK, F. E. Dynamics of residue decomposition and soil organic matter turnover. In: SOIL microbiology and biochemistry. $2^{\text {nd }} e d$. San Diego: Academic, 1996. p. 158-179.

SCHOMBERG, H. H.; STEINER, J. L. Nutrient dynamics of crop residues decomposing on a fallow no-till soil surface. Soil Science Society of America Journal, Madison, v. 63, n. 3, p. 607-613, 1999.

SOEST, P. J. van; WINE, R. H. Use of detergents in analysis of fibrous feeds -4 : determination of plant cell-wall constituents. Journal of the Association of Official Analytical Chemists, Gaithersburg, v. 50, n. 1, p. 50-57, 1967.

TEDESCO, M. J.; GIANELLO, C.; BISSANI, C. A.; BOHNEN, H.; VOLKWEISS, S. J. Análises de solo, plantas e outros materiais. Porto Alegre: UFRGS , 1995. 174 p. (Boletim Técnico, 5)

WHITE, R. E.; AYOUB, A. T. Decomposition of plant residues of variable $\mathrm{C} / \mathrm{P}$ and the effect on phosphate availability. Plant Soil, Dordrecht, v. 74, n. 2, p. 164-173, 1983.

WIEDER, R. K.; LANG, G. E. A critique of the analytical methods used in examining decomposition data obtained from litter bags. Ecology, Washington, v. 63, n. 6 , p. 1636-1642, 1982. 